- five-day search, 10 kilometres from the conference venue, and police opened a homicide investigation.

On a web page posted last week by the Max Planck Institute of Molecular Cell Biology and Genetics in Dresden, Germany, where Eaton worked, colleagues describe her as a worldrenowned developmental biologist of singular passion, depth and breadth.

Eaton studied how particular molecules control embryonic development in fruit flies, and she had been scheduled to give the meeting's plenary lecture two days after the date of her disappearance.

\section{'DEEP, DEEP SORROW'}

"Her curiosity and enthusiasm for discovery was infectious," write her lab members. "She was our leader, our role-model, our mentor, our friend," they say. "Her sudden and tragic death has left us stunned and enveloped in deep, deep sorrow."

Colleagues also praised the work-life balance she achieved - she was the mother of two boys, as well as a talented musician and a black belt in tae kwon do.

"She worried that it was impossible to give both her science and her family her all," writes her sister. "With a deep sensitivity and compassion, she somehow made us all a priority."

Meeting attendees were thrown into turmoil when they realized Eaton was missing, says François Leulier, a molecular geneticist at the Institute of Func-

tional Genomics of Lyon, France, who was also a conference plenary speaker. They had seen Eaton playing the piano on the afternoon that she disappeared, and

\section{"With her} extensive scientific culture, she brought a richness to every meeting."

thought little of the fact that she did not attend the evening session that day, he says.

There were no lectures the following afternoon and some attendees, including the meeting organizers, began to discuss Eaton's absence. "We hoped that she had joined the conference excursion, but when they returned for the evening session without her we were really worried," says Leulier.
The group of attendees went to her room and found the wake-up alarm on her smartphone still ringing, indicating that she had not been there overnight. They drove straight to the police to report her missing, Leulier says, then at daybreak they divided into search groups and began to comb the shore and trails. Later that day, the police asked the scientists to remain at the conference centre to allow them to take charge of the search.

Leulier says he knew Eaton from the international conference circuit, where she was renowned for driving lively discussions. "With her extensive scientific culture, she brought a richness to every meeting," he says. "She asked probing questions on every subject in such a subtle, empathic and positive way."

During her career, Eaton had also been a staff scientist at the European Molecular Biology Laboratory (EMBL) in Heidelberg, Germany. "The EMBL community is in shock and mourning," says a tribute web page posted by the laboratory. Cell biologist Kai Simons, Eaton's mentor at EMBL, writes that "she represented a modern Renaissance scientist in the sheer scope of her activities"..

\title{
Japanese spacecraft probes asteroid's guts for first time
}

\section{Hayabusa2 touched down on Ryugu to collect material from beneath the surface.}

\section{BY DAVIDE CASTELVECCHI}

$\int$ apan's Hayabusa2 asteroid mission has performed the last major act in its saga of space exploration. At 10:18 a.m. Tokyo time on 11 July, the spacecraft descended on the asteroid Ryugu for the second time this year,
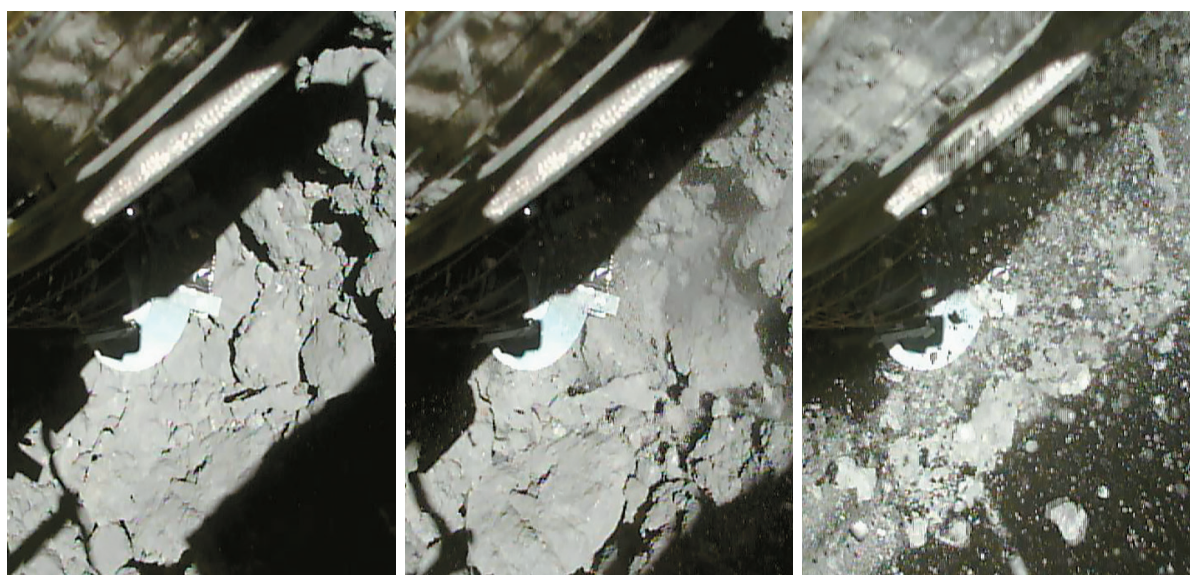

Images taken by Hayabusa 2 as it descended towards the asteroid Ryugu.

to collect material from a crater it gouged out in April by striking the body's surface with a pellet. If the collection was successful something that the mission team will not know for a while - it will be the first time in history that a mission has gathered material from an asteroid's innards.
The probe collected a sample from Ryugu's surface in February. After it returns its booty to Earth next year, scientists will be able to compare the composition of material from the two touchdown sites. That could reveal how exposure to the rigours of space, and in particular solar heating, solar wind and cosmic rays, affected the chemistry on the surface.

"This is a cornucopia of a mission," says Lucy McFadden, a planetary astronomer at NASA's Goddard Space Flight Center in Greenbelt, Maryland.

Hayabusa2 arrived at Ryugu in June 2018. It deployed landers that took magnetic, chemical and other measurements and sent pictures back. The spacecraft completed its first touchdown in February this year and then, in April, it shot a projectile that produced a 10 -metrewide crater, uncovering material under the asteroid's surface. Later this year, Hayabusa 2 will turn back to Earth, where by the end of 2020 it is expected to deliver its samples for analysis.

In its latest move, Hayabusa2 aimed for a spot just outside the crater, rather than 
descending inside the crater itself, which would have been "rather risky", mission manager Makoto Yoshikawa of the Japan Aerospace Exploration Agency (JAXA) Institute of Space and Aeronautical Science in Sagamihara told Nature.

"If you're going into a depression, then you have to worry about things like the solar panels sticking out" and potentially colliding with the surface, says Harold Connolly, a cosmochemist at Rowan University in Glassboro, New Jersey, and a co-investigator on the mission team. He is also working on NASA's OSIRIS-REx mission, which is exploring a similar body - called Bennu and plans to collect material from its surface next year. The two missions exchange information and collaborate, in part by sharing staff.

The 1-kilometre-wide Ryugu is what scientists call a rubble-pile asteroid: a collection of rocks and dust held together loosely by gravity. Its low density - only slightly higher than that of liquid water suggests that it is mostly empty space, and that it has accumulated from debris produced by a collision of other bodies, Connolly says.

Suction does not work in the vacuum of space, and Ryugu has almost no gravity. So the team devised an original technique that allows the spacecraft to pick up material while bouncing on the surface, without actually landing. The method involved loosening material and catching it in a horn (see 'Asteroid treasure').

The goal is to bring back a total of around a gram of material. But the team will have to wait until the probe returns to Earth to open the chambers and see what's inside. While

\section{ASTEROID TREASURE}

With its final major manoeuvre, Japan's spacecraft Hayabusa2 is aiming to become the first probe ever to collect material from beneath the surface of an asteroid.

1 Hayabusa2 gently descends towards
the surface of asteroid Ryugu.

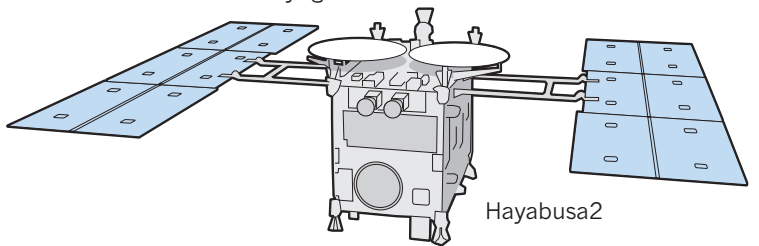

2 The craft doesn't land, but hovers near a crater it created in April, exposing subsurface material.

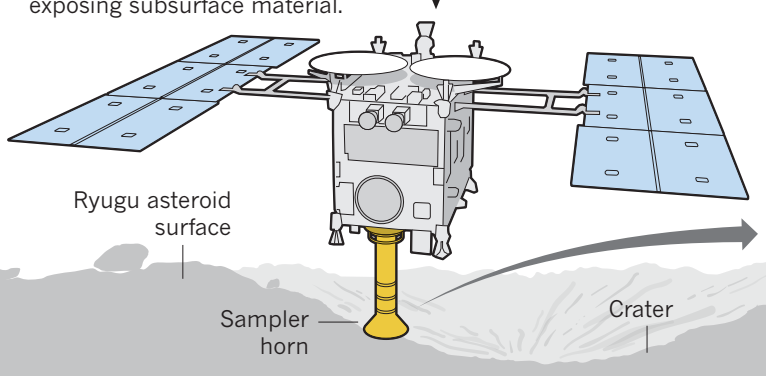

\section{4}

Fragments of material ricochet inside the horn and are captured in one of three collection chambers.

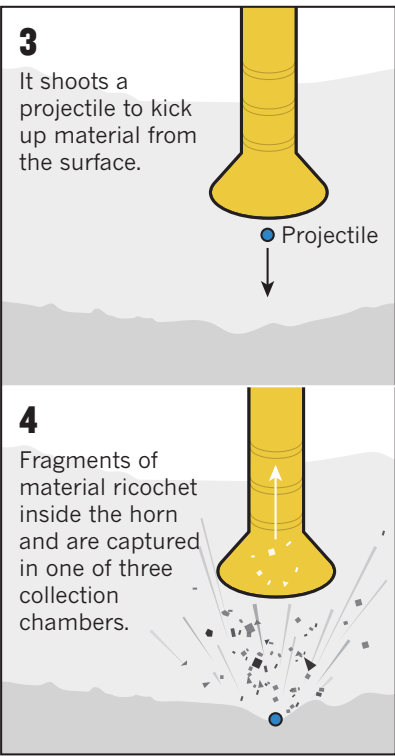

Hayabusa2 is in space, mission control has no way of knowing how much material has been collected in each touchdown operation, Yoshikawa says.

Physicists hope that the materials will help to solve asteroid mysteries - for instance, it's not clear why Ryugu is so dark. It is among the least reflective bodies in the Solar System, darker than any known meteorite, and the material exposed at the bottom of the freshly dug crater is darker still. Researchers with JAXA are keen to find out whether the April impact itself made the material darker, or whether the crater's colour is typical of Ryugu's composition and the surface has been lightened by solar radiation.

Ryugu's surface is also strewn with an unusual number of boulders - more per unit surface area than any asteroid explored so far, according to a paper the mission scientists published in May (T. Michikami et al. Icarus 331, 179-191; 2019). This makes the approach and touchdown particularly hazardous for Hayabusa2, especially given that the craft has to operate autonomously owing to its large distance from Earth..

\section{AI beats professionals at six-player poker}

\section{Triumph in complex variant of game brings bots closer to solving thorny real-world problems.}

\section{BY DOUGLAS HEAVEN}

$\mathrm{M}$ achines have raised the stakes once again. A superhuman poker-playing bot called Pluribus has beaten top human professionals at six-player no-limit Texas hold 'em poker, the most popular variant of the game. It is the first time that an artificial-intelligence (AI) program has beaten elite human players at a game with more than two participants (N. Brown and T. Sandholm Science http://doi.org/c766; 2019).
"While going from two to six players might seem incremental, it's actually a big deal," says Julian Togelius at New York University in New York City, who studies games and AI. "The multiplayer aspect is something that is not present at all in other games that are currently studied."

The team behind Pluribus had already built an AI, called Libratus, that had beaten professionals at two-player poker. It built Pluribus by updating Libratus and created a bot that needs much less computing power to play matches. In a 12-day session with more than 10,000 hands, it beat 15 leading human players. "A lot of AI researchers didn't think it was possible to do this" with our techniques, says Noam Brown at Carnegie Mellon University in Pittsburgh, Pennsylvania, and Facebook AI Research in New York City, who developed Pluribus with his Carnegie colleague Tuomas Sandholm.

Other AIs that have mastered human games - such as Libratus and DeepMind's Go-playing bots - have shown that they are unbeatable in two-player zero-sum matches. In these scenarios, there is always one winner and one loser, and game theory offers a well-defined best strategy - use it and you can't lose.

But game theory is less helpful for scenarios involving multiple parties with competing interests and no clear win-lose conditions - which reflect most real-life challenges. By solving multiplayer poker, Pluribus lays the foundation for future AIs to tackle complex problems of this sort, says Brown. He thinks that the success is a step towards applications such as automated negotiations, better fraud detection and self-driving cars.

To tackle six-player poker, Brown 